\title{
RELAÇÕES MORFOMÉTRICAS DE Ocotea odorifera (Vell.) Rohwer
}

\author{
Morphometric relations of Ocotea odorifera (Vell.) Rohwer
}

\author{
Enrique Orellana $^{a}$, Alexandre Bernardi Koehler ${ }^{b}$ \\ a Engenheiro Florestal, Mestrando em Ciências Florestais pela UNICENTRO, Irati, Paraná - Brasil, e-mail: \\ enriqueorellana@uol.com.br

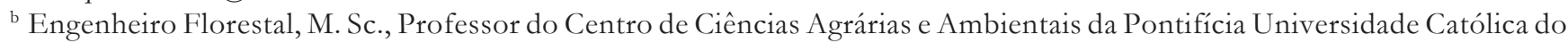 \\ Paraná, São José dos Pinhais, PR - Brasil, e-mail: alexandre.koehler@pucpr.br
}

\section{Resumo}

O objetivo do presente estudo foi avaliar a correlação dos principais índices morfométricos da copa com o diâmetro (DAP) e a altura total (HT) da canela-sassafrás (Ocotea odorifera). A coleta de dados foi feita na Fazenda Experimental Gralha Azul, da Pontifícia Universidade Católica do Paraná, no município de Fazenda Rio Grande, PR. Ao todo foram medidas 50 árvores, com o DAP $>10 \mathrm{~cm}$. Variáveis como comprimento da copa (L), diâmetro de copa (DC), proporção de copa (PC), índice de saliência (IS), índice de abrangência (IA), formal de copa (FC) e grau de esbeltez (GE) foram correlacionadas com o diâmetro e a altura total. Os resultados indicaram que existem correlações positivas do DAP com os índices HT, DC, L, IS, IA e GE. Para a altura total (HT) foram encontradas correlações somente com DC e L.

Palavras-chave: Morfometria; Sassafrás; Fazenda experimental Gralha Azul.

\begin{abstract}
The objective of the present work was to evaluate the correlations between the main crown morphometric indexes with the DBH and total height of Ocotea odorifera. The data was collected in the Gralha Azul Experimental Farm, of the Pontifical Catholic University of Parana, at the Municipality of Fazenda Rio Grande, PR. Fifty trees with DBH $>10 \mathrm{~cm}$ were measured. Variables such as crown length $(L)$, crown diameter (DC), crown proportion (PC), salience index (IS), range index (IA), crown formal (FC), and slenderness rate (GE) were correlated with diameter (DBH) and total height (HT). The results indicate that exist positive correlations between DBH with the $H T, D C, L, I S, I A$ and GE indexes. The HT variable was correlated only with DC and L.
\end{abstract}

Keywords: Morphometry; Sassafras; Gralha Azul experimental farm. 


\section{INTRODUÇÃO}

O principal recurso econômico oriundo das florestas é a madeira. Por isso, a maioria dos trabalhos de inventário florestal tem sido conduzida visando a quantificação da biomassa lenhosa aproveitável para serraria, papel e celulose e lenha. Mas, algumas espécies, como é o caso do sassafrás Ocotea odorifera (Vell.) Rohwer, fornecem produtos alternativos, nesse caso um óleo essencial de elevado valor comercial.

Santos (1987) afirma que da canela sassafrás, ou sassafrás (Ocotea odorifera) se extrai um óleo precioso, denominado safrol e utilizado para vários fins, principalmente como fixador de perfume. Este valioso produto é obtido da destilação não só do tronco, mas também da casca, folhas e raízes (SANTOS, 1987). Isto possibilita que planos de manejo para o aproveitamento comercial do óleo sejam elaborados aos moldes do que se aplica na exploração das folhas de erva-mate (Ilex paraguariensis St. Hill.) em florestas nativas (PUCPR, 2005).

Como a espécie ocorre em distintas formações florestais brasileiras, na Floresta Estacional Semidecidual, na Floresta Ombrófila Densa e na Floresta Ombrófila Mista (CARVALHO, 1994), a exploração das folhas para a obtenção de óleo essencial surge como uma importante alternativa de obtenção de renda com o manejo de baixo impacto na floresta nativa, valorizando estes ambientes e promovendo, consequentemente, sua conservação (PUCPR, 2005).

Para a elaboração de um modelo de exploração das folhas de sassafrás é preciso investigar a morfologia da copa da árvore e suas possíveis relações com variáveis de fácil obtenção, como o diâmetro do tronco e a altura. Este é o primeiro passo para o desenvolvimento de modelos matemáticos que estimem a biomassa foliar em função de variáveis biométricas. Além disso, é importante investigar estas relações nas diferentes posições sociológicas ocupadas pela espécie na comunidade florestal. Estes modelos auxiliam a determinação do número de árvores a serem exploradas por unidade de área, com base no volume do compartimento da biomassa de interesse, visando sempre o uso racional e sustentável do recurso.

De forma geral os pesquisadores florestais têm trabalhado na seleção, criação e ajuste de modelos matemáticos que sirvam para a predição da biomassa em função de variáveis de fácil obtenção (SANQUETTA, 2002). Segundo Pascoa et al. (2004) a estimativa da biomassa das florestas é de enorme interesse, não só ao nível científico como prático. Esses autores explicam que os estudos de biomassa florestal são feitos com propósitos variados, como, por exemplo, para a quantificação da reciclagem de nutrientes, para a quantificação da biomassa para fins energéticos, como base de informação para estudos de fixação e seqüestro de carbono, entre outros. Em qualquer dos casos referidos, estes estudos são de grande importância para a tomada de decisões de gestão dos recursos florestais.

A distribuição da biomassa vegetal nos indivíduos tem uma porção aérea e uma subterrânea. A proporção em que se distribui essa biomassa vegetal não é constante para todos os indivíduos, inclusive dentro da mesma espécie, e está sujeita a fatores diversos. Entre estes, pode-se mencionar as características genéticas das espécies, a idade dos indivíduos e as condições do ambiente (CASTRO; KAUFFMAN, 1998; KUNTSCHIK, 2004).

O estudo das relações morfométricas de uma espécie florestal fornece os subsídios básicos para estimativas da biomassa de copa. Variáveis como altura total da árvore, altura de copa e o diâmetro de copa podem ser correlacionados com a altura total e o diâmetro à altura do peito (DAP). Durlo e Denardi (1998) pesquisaram a espécie nativa Cabralea canjerana, no Rio Grande do Sul, e denotaram haver correlações entre diferentes variáveis em função do DAP e da altura total, como: Proporção de Copa (PC), Índice de Saliência (IS), Índice de Abrangência (IA), Formal de Copa (FC) e Grau de Esbeltez (GE).

A razão entre o comprimento de copa e altura total da copa em porcentagem também é conhecido como a Proporção de Copa (PC) e indica a fração (\%) que a copa abrange em relação à árvore. Quanto maior a porcentagem de copa, tanto mais vital e produtiva é a árvore (DURLO; DENARDI, 1998).

O Índice de Saliência (IS) é a relação entre o diâmetro de copa e o DAP e indica quantas vezes o diâmetro de copa é maior que o DAP. Quando se prevê o manejo de um povoamento não pela sua idade, mas pelo diâmetro atingido por seus componentes, pode-se determinar o número máximo de árvores por unidade de área, com o cálculo do índice de saliência, se existir uma correlação significativa com o DAP. Portanto, este índice pode ser usado como indicador de desbaste, determinando, a qualquer tempo, o espaço a ser liberado ao redor de uma árvore selecionada, para que esta cresça sem concorrência (DURLO; DENARDI, 1998).

Rev. Acad., Ciênc. Agrár. Ambient., Curitiba, v. 6, n. 2, p. 229-237, abr./jun. 2008 
A razão entre o diâmetro de copa e a altura total da árvore também é conhecida como Índice de Abrangência (IA). Este índice pode ser considerado para a avaliação do momento de desbaste, caso haja uma alta correlação entre estas variáveis (DURLO; DENARDI, 1998).

O Formal de Copa (FC) é a relação entre o diâmetro e a altura de copa. Quanto menor o formal de copa, melhor é a produtividade da árvore. Este índice também funciona como critério para a marcação de desbastes (TONINI; ARCO-VERDE, 2005).

O Grau de Esbelteza (GE) é a relação entre a altura total da árvore e o DAP. Uma relação superior a 1 indica que o crescimento em diâmetro é reduzido em relação à altura (TONINI; ARCOVERDE, 2005).

O objetivo do presente estudo foi avaliar as possíveis correlações entre variáveis biométricas e morfométricas do sassafrás em diferentes posições sociológicas nas quais a árvore ocorre em um remanescente da Floresta Ombrófila Mista, com a finalidade de fornecer parte dos subsídios necessários à elaboração de um plano de manejo de exploração das folhas e ramos finos de sassafrás para a produção de óleo essencial.

\section{MATERIAL E MÉTODOS}

A coleta de dados foi efetuada em parte de um remanescente bem conservado da Floresta Ombrófila Mista, no município de Fazenda Rio Grande, estado do Paraná, a cerca de $21 \mathrm{~km}$ da cidade de Curitiba. A área está inserida na Fazenda Experimental Gralha Azul, propriedade da Associação Paranaense de Cultura, entidade mantenedora da Pontifícia Universidade Católica do Paraná. O clima predominante na região é o Cfb, com altitudes variando entre 870 metros e 920 metros.

Segundo Bognola et al. (2003), no levantamento dos solos encontrados na Fazenda Experimental Gralha Azul, nas áreas onde predomina a floresta primária parcialmente alterada, ocorrem o Cambissolo Húmico Tb distrófico dos tipos Latossólico Típico e Gleico, e Cambissolo Háplico Tb distrófico típico.

As árvores medidas foram aleatoriamente selecionadas na parcela número 1, demarcada por ocasião dos trabalhos de campo do projeto Sassafrás: Bioecologia e Uso Sustentável, desenvolvido pelo Centro de Ciências Agrárias e Ambientais da PUCPR, nos anos de 2003 e 2004. Nesse período, todos os indivíduos de sassafrás adultos foram localizados, numerados e mensurados, em todas as áreas com cobertura florestal na Fazenda Experimental Gralha Azul (PUCPR, 2005).

Para o cálculo da suficiência amostral selecionou-se a variável DAP como base para a determinação do número mínimo de amostras necessárias para abranger a variabilidade dimensional da população, com a seguinte equação (1), conforme Péllico Netto e Brena (1997):

$$
n=\frac{1}{\frac{\epsilon^{2}}{t^{2} s_{x}^{2}}+\frac{1}{N}}
$$

Onde:

$n$ - número de unidades amostrais a ser medido;

$N$ - número potencial de unidades amostrais;

$t$ - valor de $t$ de student tabelado;

LE - limite de erro da amostragem admitido em \%;

$\bar{y}$ - média aritmética dos valores observados;

- variância;

$\in=L E \cdot \bar{y}$ - erro admissível em valor absoluto da variável de interesse. 
Ao total foram medidas 50 árvores, com o critério mínimo de inclusão DAP $>10 \mathrm{~cm}$. De cada uma foram medidos os 4 raios de projeção da copa, nos sentidos N, S, L e O. Anotou-se ainda, na ficha de campo, a posição sociológica, sendo 1 para árvores de dossel (dominantes), 2 para árvores do estrato co-dominante e 3 para árvores dominadas, além das variáveis DAP, altura total e altura do ponto de inserção da copa.

Com base nos valores medidos de DAP, altura total (Ht), altura de inserção de copa (IC) e raios de projeção da copa, foi possível calcular o diâmetro de copa (DC), o índice de saliência (IS), o índice de abrangência (IA), a proporção de copa (PC) e o formal de copa (FC).

A equação utilizada para o cálculo do diâmetro de copa foi o modelo logarítmico genérico (Equação 2). Este modelo foi ajustado e validado por Tonini e Arco-Verde (2005) para Hymenaea courbaril.

Para a correção da discrepância logarítmica do modelo testado para a estimativa do diâmetro de copa aplicou-se o fator de correção de Meyer, dado pela equação 3.

$$
F M=e^{0,5 \cdot S_{y x}^{2}}
$$

Onde: $\quad e$ - base do logaritmo natural $(l n)$; $S_{y x}$ - erro padrão da estimativa (m)

O índice de Schlaegel foi calculado segundo a equação 4, tendo como base de dados os pares DAP e DC. Este indicador de ajuste (IA) mostra o quanto de alteração da variável dependente é explicado pelas variáveis independentes. O IA varia de 0 a 1 e o quanto mais próximo deste valor melhor é o ajuste do modelo. Este índice é aplicado somente para os modelos com transformação logarítmica de dados.

$$
I A=1-\frac{\text { SQres }}{S Q T}
$$

Onde:

SQres - Soma dos quadrados do resíduo

$S Q T$ - Soma dos quadrados total

\section{RESULTADOS E DISCUSSÃO}

O número mínimo de amostras necessárias, calculado com base na variável DAP, foi de 46 árvores, considerando-se indivíduos com diâmetro a altura do peito maior que $10 \mathrm{~cm}$.

TABELA 1 - Coeficientes e indicadores de ajuste para o modelo de estimativa do diâmetro de copa (DC) testado para Ocotea odorifera na Fazenda Experimental Gralha Azul, PR, 2006

Table 1 - Parameters and adjustment indicators for the crown diameter (DC) estimation model, tested for Ocotea odorifera, at the Gralha Azul Experimental Farm, PR, 2006

\begin{tabular}{ccccccc}
\hline EQUAÇÃO & \multicolumn{4}{c}{ COEFICIENTES } & \multicolumn{4}{c}{ AJUSTE } \\
\hline In DC = bO+b1 DAP & $b_{0}$ & $b_{1}$ & R2 & IA & $\mathrm{S}_{\mathrm{yx}}$ & $\mathrm{F}$ \\
& 1,221805 & 0,036373 & 0,579063 & 0,9558 & 0,225223 & 68,4069 \\
\hline
\end{tabular}

Rev. Acad., Ciênc. Agrár. Ambient., Curitiba, v. 6, n. 2, p. 229-237, abr./jun. 2008 
De acordo com o Índice de Schlaegel calculado de 0,95, o modelo utilizado se ajusta satisfatoriamente aos dados testados (Tabela 1). A análise gráfica dos resíduos corrobora com esta afirmativa, onde não se observa nenhuma discrepância sistemática entre os resíduos e há homogeneidade na variância, exceto por duas observações de árvores mais finas, cujas estimativas excedem os 50\% de desvio em relação à linha média de regressão (Figura 1).

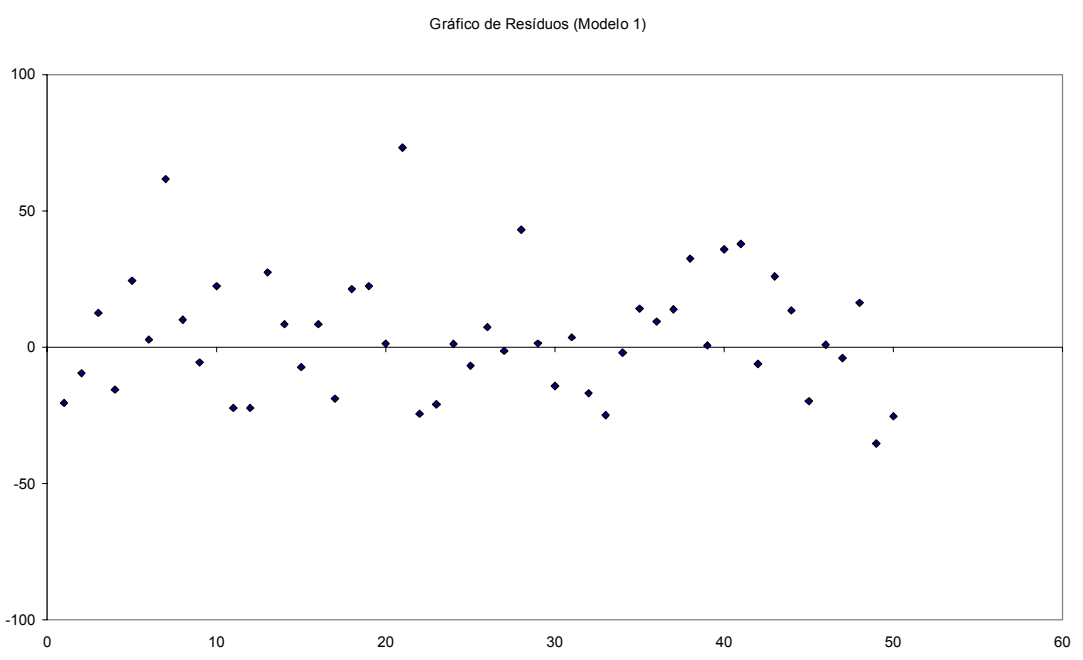

FIGURA 1 - Gráfico dos resíduos para o modelo ajustado para diâmetro de copa (DC) em função do DAP, para Ocotea odorifera, na Fazenda Experimental Gralha Azul, PR, 2006

Figure 1 - Residuals graphic of the adjusted model for crown diameter as function of the DBH, for Ocotea odorifera, at the Gralha Azul Experimental Farm, PR, 2006

Para as árvores amostradas em campo, o DAP médio foi de 18,68 cm, sendo que a árvore de maior diâmetro apresenta $43 \mathrm{~cm}$ e se encontra na posição sociológica 1 (dominante). As árvores de menor diâmetro foram aquelas de $10 \mathrm{~cm}$ de diâmetro, valor mínimo avaliado no inventário e encontrada nas posições sociológicas 2 e 3.

A altura total (HT) média de todas as árvores foi de 10,58 $\mathrm{m}$ tendo-se como valor máximo $18 \mathrm{~m}$ e valor mínimo de $6 \mathrm{~m}$, encontrados nas posições sociológicas 1 e 3 respectivamente (Tabela 2).

TABELA 2 - Parâmetros biométricos e morfométricos de Ocotea odorifera na

Fazenda Experimental Gralha Azul, PR, 2006

Table 2 - Biometric and morphometric parameters of Ocotea odorifera at the Gralha Azul Experimental Farm, PR, 2006

\begin{tabular}{|c|c|c|c|c|c|c|c|c|c|c|}
\hline & \multicolumn{6}{|c|}{ Biometria } & \multicolumn{4}{|c|}{ Morfometria } \\
\hline & $\begin{array}{l}\text { DAP } \\
(\mathrm{cm})\end{array}$ & $\begin{array}{l}\mathrm{HT} \\
(\mathrm{m})\end{array}$ & $\begin{array}{l}\mathrm{HC} \\
(\mathrm{m})\end{array}$ & $\begin{array}{l}\mathrm{DC} \\
(\mathrm{m})\end{array}$ & $\begin{array}{l}\mathrm{AC} \\
\left(\mathrm{m}^{2}\right)\end{array}$ & $\begin{array}{l}\mathrm{PC} \\
(\%)\end{array}$ & IS & GE & IA & $\mathrm{FC}$ \\
\hline Médio & 18,68 & 10,58 & 4,72 & 7,1 & 5,9 & 55,2 & 39,2 & 0,6 & 0,7 & 1,3 \\
\hline Máximo & 43 & 18 & 8 & 13,25 & 11 & 77,8 & 68,6 & 0,9 & 1 & 2,3 \\
\hline Mínimo & 10 & 6 & 2 & 2,76 & 2 & 30 & 24,7 & 0,3 & 0,3 & 0,4 \\
\hline $\mathrm{CV} \%$ & 39,16 & 20,65 & 32,04 & 33,97 & 31,87 & 23,30 & 23,20 & 28,13 & 24,2 & 32,7 \\
\hline
\end{tabular}

Rev. Acad., Ciênc. Agrár. Ambient., Curitiba, v. 6, n. 2, p. 229-237, abr./jun. 2008 
O diâmetro médio de copa (DC) foi de $7,1 \mathrm{~m}$, sendo que o maior diâmetro de copa encontrado foi de 13,25 m (posição sociológica 1) e o menor valor encontrado de 2,76 m (posição sociológica 2). Estudos realizados por Durlo e Denardi (1998), no Rio Grande do Sul com 165 árvores da espécie Cabralea canjerana, apontam um valor de diâmetro de copa médio de 4,30 m. O menor valor encontrado para a espécie foi de $1 \mathrm{~m}$ e o maior valor $10,40 \mathrm{~m}$.

A proporção média de copa (PC) foi de $55,2 \%$, ou seja, $55,2 \%$ da altura total das árvores em média é ocupada pela copa. A maior proporção de copa encontrada foi de $69,23 \%$ na posição sociológica 1 (dominante). A menor proporção de copa encontrada foi de $30 \%$ na posição sociológica 3 (sub-bosque). Durlo; Dernardi (1998) encontraram valor de proporção de copa médio de 49,2\%. O valor máximo de proporção de copa encontrado por esses autores foi de $83,3 \%$ e o valor mínimo encontrado foi de $10,7 \%$.

O Índice de Saliência (IS) revela quantas vezes a copa é maior que o DAP. Neste trabalho foi verificado que a copa das árvores de Ocotea odorifera supera, em média, 39,2 vezes a dimensão do DAP. O maior valor encontrado foi de 68,5 , localizado na posição sociológica 2 e o menor valor foi de 24,72, também encontrado na posição sociológica 2. Durlo e Dernardi (1998) encontraram, para Cabralea canjerana, valores médios de índice de saliência de 21,70, sendo que o menor valor encontrado foi de 12,8 e o maior foi de 35,70. Tonini e Arco Verde (2005) encontraram valores médios de índice de saliência para a castanheira-do-brasil (Bertholletia excelsa) de 50; para a andiroba (Carapa guianensis) de 14,7; para o ipê-roxo (Tabebuia avellanedae) de 32,3 e para o jatobá (Hymenaea courbaril) de 33,1.

O índice de abrangência (IA) é a relação entre diâmetro de copa e altura total. Se um povoamento for manejado pela altura das árvores (altura-objetivo), pode-se usar este índice como critério para tomada de decisão em um plano de desbaste. O índice de abrangência médio encontrado foi de 0,7 , sendo que o maior valor encontrado foi de 1 e o menor foi de 0,3.

O valor médio formal de copa (FC) foi de 1,3. O valor máximo encontrado foi de 2,31 e o menor valor de 0,39 , ambos encontrados na posição sociológica 3. Valores baixos indicam árvores com copas esbeltas, enquanto valores de copa altos indicam copas achatadas. Os valores obtidos indicam que existe uma grande amplitude de variação entre os indivíduos. O mesmo foi verificado em estudos realizados por Durlo e Dernardi (1998, p.63) com a Cabralea canjerana onde eles obtiveram valores médios de 0,74 com variações entre 0,29 e 3,33.

Nenhuma árvore apresentou grau de esbeltez (GE) superior a 1. O valor médio encontrado foi de 0,62. O valor máximo encontrado foi 0,91 e o mínimo 0,34.

A seguir estão representados os parâmetros biométricos das árvores em diferentes posições sociológicas (Tabela 3).

TABELA 3 - Parâmetros biométricos de Ocotea odorifera, nas posições sociológicas 1 (dominante); 2 (codominantes) e 3 (dominadas) na Fazenda Experimental Gralha Azul, PR, 2006

Table 3 - Biometric parameters of Ocotea odorifera, in the sociological positions 1 (dominant), 2 (co-dominant) and 3 (dominated), at the Gralha Azul Experimental Farm, PR, 2006

\begin{tabular}{|c|c|c|c|c|c|c|c|c|c|c|c|c|}
\hline & \multicolumn{3}{|c|}{ DAP (cm) } & \multicolumn{3}{|c|}{$\mathrm{HC}(\mathrm{m})$} & \multicolumn{3}{|c|}{ HT (m) } & \multicolumn{3}{|c|}{$\mathrm{DC}(\mathrm{m})$} \\
\hline & 1 & 2 & 3 & 1 & 2 & 3 & 1 & 2 & 3 & 1 & 2 & 3 \\
\hline média & 27,14 & 15,9 & 14,67 & 5,42 & 4,57 & 4,26 & 13,21 & 10,14 & 9,11 & 9,52 & 7,01 & 4,94 \\
\hline máximo & 43 & 28 & 21 & 8 & 7 & 8 & 18 & 13 & 12 & 13,25 & 10,27 & 6,94 \\
\hline mínimo & 15 & 10 & 10 & 4 & 2 & 2 & 11 & 8 & 6 & 5,97 & 3,96 & 2,76 \\
\hline $\mathrm{CV} \%$ & 26,87 & 27,4 & 21,97 & 22,52 & 24,53 & 47,26 & 13,83 & 12,58 & 17,82 & 21,76 & 24,07 & 24,31 \\
\hline
\end{tabular}


As Tabelas 4 e 5 contêm os dados referentes aos parâmetros morfométricos das árvores em diferentes posições sociológicas.

TABELA 4 - Índices morfométricos de Ocotea odorifera, nas posições sociológicas 1 (dominante); 2 (co-dominantes) e 3 (dominadas), na Fazenda Experimental Gralha Azul, PR, 2006

Table 4 - Morphometric indexes of Ocotea odorifera, in the sociological positions 1 (dominant), 2 (codominant) and 3 (dominated), at the Gralha Azul Experimental Farm, PR, 2006

\begin{tabular}{lccccccccc}
\hline & \multicolumn{3}{c}{ Altura de copa $(\mathbf{m})$} & \multicolumn{3}{c}{ Proporção de copa (m) } & \multicolumn{3}{c}{ Índice de Saliência } \\
& $\mathbf{1}$ & $\mathbf{2}$ & $\mathbf{3}$ & $\mathbf{1}$ & $\mathbf{2}$ & $\mathbf{3}$ & $\mathbf{1}$ & $\mathbf{2}$ & $\mathbf{3}$ \\
\hline média & 7,57 & 5,57 & 4,73 & 58,00 & 54,73 & 53,21 & 36,18 & 45,14 & 33,81 \\
máximo & 11,00 & 8,00 & 7,00 & 69,23 & 75,00 & 77,77 & 47,8 & 68,55 & 42,23 \\
mínimo & 5,00 & 3,00 & 2,00 & 38,46 & 33,33 & 30,00 & 26,74 & 24,72 & 25,09 \\
\hline CV\% & 23,00 & 24,46 & 33,37 & 15,72 & 19,96 & 33,57 & 21,04 & 20,23 & 14,35 \\
\hline
\end{tabular}

Pela análise da Tabela 4 percebe-se que no primeiro estrato os valores de altura e proporção de copa são superiores aos calculados para as árvores que ocupam os estratos inferiores. No entanto, o maior índice de saliência foi calculado para as árvores que ocupam o estrato co-dominante. Isto mostra que as árvores deste estrato possuem copas mais largas em relação ao diâmetro do tronco. A incidência de luz neste estrato é menor do que no estrato superior e, neste caso, parece haver uma espécie de compensação morfométrica, uma vez que as árvores dominantes possuem copas, de maneira geral, mais alongadas.

TABELA 5 - Índices morfométricos de Ocotea odorifera, nas posições sociológicas 1 (dominante);

2 (co-dominantes) e 3 (dominadas), na Fazenda Experimental Gralha Azul, PR, 2006

Table 5 - Morphometric indexes of Ocotea odorifera, in the sociological positions 1 (dominant), 2 (co-dominant) and 3 (dominated), at the Gralha Azul Experimental Farm, PR, 2006

\begin{tabular}{|c|c|c|c|c|c|c|c|c|c|}
\hline & \multicolumn{3}{|c|}{ Índice de Abrangência } & \multicolumn{3}{|c|}{ Formal de Copa } & \multicolumn{3}{|c|}{ Grau de Esbeltez } \\
\hline & 1 & 2 & 3 & 1 & 2 & 3 & 1 & 2 & 3 \\
\hline média & 0,74 & 0,69 & 0,56 & 1,31 & 1,31 & 1,17 & 0,50 & 0,67 & 0,64 \\
\hline máximo & 1,04 & 1,03 & 0,87 & 2,18 & 2,28 & 2,32 & 0,93 & 0,92 & 0,91 \\
\hline mínimo & 0,48 & 0,44 & 0,28 & 0,84 & 0,80 & 0,39 & 0,34 & 0,39 & 0,38 \\
\hline $\mathrm{CV} \%$ & 23,12 & 21,77 & 26,86 & 30,33 & 29,30 & 43,68 & 29,15 & 21,83 & 27,83 \\
\hline
\end{tabular}

No caso do índice de abrangência (IA) o maior valor foi calculado para as árvores dominantes. Em relação ao formal de copa (FC) os valores médios para os estratos dominantes e co-dominantes foram os mesmos, mas inferiores no estrato dominado. Segundo Durlo e Denardi (1998), quando menor este valor maior a produtividade da árvore e, neste caso, há uma indicação que as árvores dominadas, certamente mais jovens, estão crescendo mais do que aquelas que já atingiram os estratos superiores. 
No caso do grau de esbeltez (GE) não foram observados valores superiores a 0,50 e, neste caso, aparentemente, não há a necessidade de desbastes, pois conforme Tonini e Arco-Verde (2005), quando este valor é superior a 1 indica-se o tempo para intervenções silviculturais na estrutura da floresta para favorecer uma espécie de interesse, em relação ao crescimento diamétrico e, por consequência, à ampliação da expansão horizontal da copa.

A correlação entre DAP e HT das árvores com as variáveis morfométricas estão descritas na Tabela 6.

TABELA 6 - Correlações de Pearson entre o DAP e a HT e as variáveis morfométricas de Ocotea odorifera, na Fazenda Experimental Gralha Azul, PR, 2006

Table 6 - Pearson correlation coefficients between DBH and HT and the morphometric variables of Ocotea odorifera, at the Gralha Azul Experimental Farm, PR, 2006

\begin{tabular}{ccccccccc}
\hline Variáveis & HT & DC & L & PC & IS & IA & FC & GE \\
\hline DAP & $0,663^{*}$ & $0,813^{* *}$ & $0,574^{*}$ & 0,179 & $-0,366^{*}$ & $0,502^{*}$ & 0,238 & $0,755^{*}$ \\
HT & - & $0,627^{*}$ & $0,733^{*}$ & 0,105 & $-0,038$ & 0,073 & $-0,900$ & $-0,870$ \\
\hline
\end{tabular}

*Correlação significativa ao nível de 0,05 .

** Correlação significativa ao nível de 0,01 .

Segundo os valores da Tabela 6, há correlações positivas do DAP com a altura total da árvore, o diâmetro de copa, o comprimento de copa e o índice de abrangência, indicando que eles crescem à medida que se aumenta o DAP. A correlação negativa do DAP com o índice de abrangência indica que este decresce com o aumento do DAP.

A respeito da altura total da árvore e as variáveis morfométricas, observou-se uma correlação significativa e positiva apenas com o diâmetro e comprimento de copa, o que indica que este tende a aumentar à medida que a altura da árvore aumenta.

\section{CONCLUSÕES}

Por meio da análise dos dados obtidos no presente estudo pode-se concluir que:

- existe uma maior correlação das características morfométricas com a variável DAP do que com a altura total (HT);

- dentre as características morfométricas correlacionadas com a altura total, apenas o diâmetro de copa (DC) e o comprimento de copa (L) apresentaram correlações significativas entre si;

- quando as características morfométricas foram correlacionadas com o DAP, observouse haver uma correlação positiva com o diâmetro de copa (DC), comprimento de copa (L), índice de saliência (IS), índice de abrangência (IA) e grau de esbeltez (GE);

- as características morfométricas variam quando analisadas separadamente em cada posição sociológica ou estrato; o que indica que a morfometria da copa varia conforme o espaço ocupado pela planta no espaço dimensional da floresta. 


\section{AGRADECIMENTOS}

Os autores expressam seus agradecimentos ao Professor Dr. Sylvio Péllico Netto, do Centro de Ciências Agrárias e Ambientais da PUCPR, coordenador do projeto Sassafrás: Bioecologia e Uso Sustentável, pelo incentivo e apoio para o desenvolvimento desta pesquisa.

\section{REFERÊNCIAS}

BOGNOLA, I. A. et al. Levantamento pedológico semidetalhado da Fazenda Experimental Gralha Azul, município de Fazenda Rio Grande - PR. Curitiba: PUCPR, 2003.

CARVALHO, P. E. R. Espécies florestais brasileiras: recomendações silviculturais, potencialidades e uso da madeira. Brasília: Embrapa-SPI, 1994.

CASTRO, E. A.; KAUFFMAN, J. B. Ecosystem structure in the Brazilian cerrado: a vegetation gradient of aboveground biomass, root biomass and consumption by fire. Journal of Tropical Ecology, Costa Rica, v. 14, p. 263-283, 1998.

DURLO, M. A; DENARDI, L. Morfometria de Ocotea odorifera em mata secundária nativa do Rio Grande do Sul. Ciência Florestal, Santa Maria, v. 8, n. 1, p. 55-66, 1998.

KUNTSCHIK, G. Estimativa da biomassa vegetal lenhosa em cerrado por meio de sensoriamento remoto óptico e de radar. 2004. 154 f. Tese (Doutorado em Ciências) - Instituto de Biociências da Universidade de São Paulo, São Paulo, 2004.

PASCOA, F. et al. Estabelecimento simultâneo de equações de biomassa para o Pinheiro Bravo. In: SIMPÓSIO IBEROAMERICANO SOBRE GESTIÓN Y ECONOMIA FORESTAL, 2., 2004. Barcelona. Anais... Barcelona: SIGEF, 2004.

PÉLLICO NETTO, S.; BRENA, D. A. Inventário florestal. Curitiba: Edição do autor, 1997. 316 p.

PONTIFÍCIA UNIVERSIDADE CATÓLICA DO PARANÁ. Projeto Sassafrás: Bioecologia e uso sustentável. Edital FNMA/PROBIO 04/2001. CHAMADA I. São José dos Pinhais: FNMAI/ PROBIO, 2005.

SANQUETTA, C. R. Métodos de determinação de biomassa florestal. In: SANQUETTA, C. R. et al. (Ed.). As florestas e o carbono. Curitiba: [s.n.], 2002. p. 119-140.

SANTOS, E. Nossas madeiras. Belo Horizonte: Itatiaia, 1987.

TONINI, H.; ARCO-VERDE M. F. Morfologia da copa para avaliar o espaço vital de quatro espécies nativas da Amazônia. Pesquisa Agropecuária Brasileira, Brasília, v. 40, n. 7, p. 633-638, 2005.

Recebido: 28/10/2007

Received: $10 / 28 / 2007$

Aprovado: $15 / 01 / 2008$

Approved: 01/15/2008 